Acta Genet Med Gemellol 30:249-255 (1981)

The Mendel Institute/Alan R. Liss, Inc.

\title{
Vitamin C and the Common Cold: A Second MZ Cotwin Control Study
}

\author{
A.B. Carr, ${ }^{1}$ R. Einstein, ${ }^{1}$ L.Y.C. Lai, ${ }^{2}$ N.G. Martin, ${ }^{3}$ G.A. Starmer ${ }^{1}$ \\ ${ }^{1}$ Department of Pharmacology, University of Sydney, ${ }^{2}$ School of Community \\ Medicine, University of New South Wales, and ${ }^{3}$ Department of Population \\ Biology, Research School of Biological Sciences, Australian National \\ University, Canberra
}

Self-reported cold data have been analyzed for 95 pairs of identical twins aged 14-64 who took part in a double-blind trial of vitamin $\mathrm{C}$ tablets. One member of each twin pair took a tablet containing $1 \mathrm{~g}$ vitamin $\mathrm{C}$ and the other took a well-matched placebo each day for 100 days. In the total sample there was no effect of vitamin $\mathrm{C}$ in preventing colds. However, subdivision of the data showed a significant preventive effect of the placebo in the 51 pairs living together and an equal and opposite preventive effect of the vitamin $\mathrm{C}$ in the 44 pairs living apart. The placebo effect in the pairs living together may be attributed to the large proportion who wrongly perceived which treatment they were taking. The reduction of colds in the vitamin $\mathrm{C}$ group of the pairs living apart was about $20 \%$. There were significant correlations between cold symptoms reported and the personality trait of neuroticism. No side effects or substantive changes in serum biochemistry could be attributed to the vitamin $\mathrm{C}$ dose.

Key words: Vitamin C, Colds, Cotwin control study, Treatment perception, Neuroticism

It is now more than ten years since the publication of Pauling's thesis [12] that a daily intake of 1-3 $\mathrm{g}$ of vitamin $\mathrm{C}$ reduces the incidence and severity of upper respiratory infections. A number of studies [1-6,10,11,13,14,16] since then have been inconsistent in their results but, in general, have failed to show any useful prophylactic effect of vitamin $\mathrm{C}$ against the common cold. Neither has any consistent therapeutic effect been demonstrated $[2,3,8,10,15]$. Nevertheless, the efficacy of vitamin $\mathrm{C}$ in prevention and cure of the common cold is still widely believed in Australia and this paper describes a test of the hypothesis under local conditions.

Most investigations of the efficacy of drugs in humans begin by assigning subjects at random to control or treatment groups $[1-3,5,6,10,14,16]$. This is less efficient than the matched pair design which removes from consideration all the factors tending to make one group different from another, so providing a more efficient test of the treatment effect. The ultimate matched pair in human experimentation is a pair of monozygotic (MZ) twins who have been reared together. Not only is there a perfect match for age, 
sex, and family background but, perhaps most importantly, genetic constitution is identical, at least for nuclear genes.

Miller et al [11] used the cotwin control design to study the effect of vitamin $\mathrm{C}$ on cold symptoms in 44 school-aged MZ twins. They found no effects in the total sample but shorter and less severe colds in the youngest group of girls and less severe colds in the youngest group of boys. We sought to replicate their study of the effects of prophylactic doses on the common cold but with a larger sample of MZ twins in an older age group.

\section{MATERIALS AND METHODS}

Permission was obtained to approach twins enrolled on the Australian Twin Registry, which contains names of over 14,000 pairs of twins of all ages living in all parts of Australia. Pairs living in the Sydney metropolitan area, born between 1916 and 1965 and claiming to be "as alike as two peas in a pod" during childhood, were contacted and 125 pairs volunteered and were selected to begin the trial. Some pairs were excluded from taking part because of a personal or family history of gout, kidney stones, diabetes, or cancer.

Zygosity was checked by typing all twins with the following antisera (anti-A,A, B,M,N,S,s,C,c,D,E,e, $\mathrm{K}, \mathrm{k}, \mathrm{Fy}^{\mathrm{a}}, \mathrm{JK}^{\mathrm{a}}$ ) and none was excluded as a dizygotic pair.

One twin of a pair was assigned at random to the treatment group and the other to the control group. The experiment was "double-blind" in that neither the subjects nor the experimenters involved with the subjects or with the analysis of the results knew which group was which until the experiment and the analysis were completed.

The treatment group received $1 \mathrm{~g}$ of ascorbic acid per day in the form of Redoxon ${ }^{\circledR}$ tablets (Roche Products), and the control group received a placebo with the same ingredients in different proportions but with lactose substituted for ascorbic acid. Quality of matching of the active and placebo tablets was checked for both appearance and taste by triangular discrimination tests on 60 pharmacology students. Two of one kind of tablet and one of the other were presented to the subject who had to pick the odd one out. It was found that subjects could pick a difference between the appearance of the dry tablets $\left(\chi^{2}=39.7\right)$ but when these were dissolved in water they were not able to pick a difference at better than chance level $\left(\chi^{2}=0.68\right)$. Having decided on the solution that tasted different, subjects were asked to identify it as active or placebo and in fact fewer than expected by chance were able to identify the solution correctly. It was concluded that the placebo was well matched in important respects and subsequent results bore this out.

In addition to the $1 \mathrm{~g}$ tablets, both groups received multivitamin capsules containing $70 \mathrm{mg}$ vitamin $\mathrm{C}$. This was to ensure that any observed treatment effect could reasonably be attributed to the pharmacologic dose of vitamin $\mathrm{C}$ and not to alleviation of dietary deficiency. Twins were thus told that one of them was on a "high dose" of vitamin $\mathrm{C}$ and the other on a "low dose."

Twins were asked to take their $1 \mathrm{~g}$ tablet (active or placebo) and multivitamin capsule at the same time each day, for 100 days beginning June 2, 1980, thus including the worst of the winter weather. They were also asked not to take any other vitamin preparations during the course of the trial. At the start of the trial, twins were given record sheets and asked to note each day the time that they had taken their tablet and capsule and also any cold symptoms present. It was stressed that if they forgot to take the tablets on any day it was more important that we knew than that they presented a perfect, but fabricated, record. To encourage compliance the sheets were to be returned in stamped addressed envelopes each four weeks and telephone reminders were given and explanations sought if these were more than two weeks late.

The ten cold symptoms listed were sore throat, sneezing, runny nose, blocked nose, cough, headache, feverish, tiredness, muscle ache, and vomiting/diarrhea. Subjects were asked to rate their symptoms $0=$ absent, $1=$ mild, 2 = moderate, 3 = severe, and also to indicate if they took a day off from work or spent a day in bed because of an upper respiratory infection.

Before the start of the trial and also during its last three weeks, subjects gave blood specimens for serum biochemical analysis to assess any changes that could be attributed to vitamin $C$ dosage. At the second attendance twins completed the Eysenck Personality Questionnaire [9], which was scored for the personality traits extraversion, neuroticism, psychoticism, and lie. At this session twins were also asked whether they thought they had been taking the high dose or low dose of vitamin C during the trial and whether they thought they had had more or fewer colds than in previous winters.

Of the 125 pairs of twins who began the trial, we have analyzed cold data for 95 pairs. Five pairs were suspended during the trial (one kidney infection, one suspected gout, two suspected allergy, and one pregnant) 
but none of these conditions could be related to the treatments. Data from other pairs were excluded if one or both failed to return record sheets, or if the record sheets showed any evidence of being rilled in retrospectively. There is no firm indication of compliance but we have excluded data from any pairs who showed evidence of negligence in following the protocol.

A cold episode was defined by the following criteria:

1) A "cold" must have a total of at least five severity points for the cold symptoms: sore throat, sneezing, runny nose, blocked nose, cough and feverish. For the purposes of classification, headaches were considered a cold symptom only if they were present together with other cold symptoms.

2) One day could be defined as an episode if the sum of cold symptom ratings was greater than or equal to five.

3) Otherwise a cold was defined as an illness episode of two or more days duration with at least two different cold symptoms (those listed in criterion 1) present together on at least two of the days, not necessarily consecutive.

4) An episode begins following three days, each with cold symptom points less than or equal to one, and ends prior to the three days each with cold symptom ratings totaling no more than one.

5) Cold episodes already present at the start of the trial were disregarded. If this initial cold lasted for more than 14 days into the trial, the individual (and pair's) cold results were excluded from analysis, as the length of the trial for that individual would have been considerably shorter than his or her cotwin.

6) Individuals were also excluded from cold data analysis if the total number of "cold" days was greater than 60 , thus excluding people with chronic respiratory conditions such as chronic bronchitis, sinusitis, and hay fever.

Twenty-nine different variables were computed from each individual's cold data. Incidence of colds: the number of defined cold episodes experienced over the trial period. Total duration of colds: the total number of "cold-days" experienced. Average duration: the total duration divided by incidence. Total severity: the total of all severity points within cold episodes reported by each individual. Average severity: the total severity divided by the incidence of colds. Intensity (the average severity per cold day): the total severity divided by the total duration. Total severity of each of the ten symptoms: the sum of the subjects ratings for that particular symptom over the trial period. Relative severity of each symptom: the total severity of the particular symptom divided by the total severity of all symptoms. Relative severity was analyzed to see whether some symptoms are affected to a greater extent than others by administration of vitamin C. Days "off work," days "in bed," and days "off work and in bed" due to colds were also analyzed as measures of severity of the colds.

The data have been analyzed by both paired and unpaired analysis of variance [7].

\section{RESULTS}

Cold data were analyzed for 38 male and 57 female pairs of MZ twins. The mean age of the sample was 25, with a range from 14 to $64 \mathrm{yr}$, and this distribution did not differ significantly between sexes. It was found that females had significantly longer, more severe, and more intense colds than males.

The results for the six summary cold variables of the paired analyses of variance between active and placebo groups are shown for the sexes separately and for the total sample in Table 1. There are no significant differences between active and placebo groups for any of the variables in either males or females considered separately. In the combined data, the vitamin $\mathrm{C}$ treatment has a significantly shorter average duration, and relative severity of cough. The reductions are small, however, and it would be surprising not to find some significant results when so many tests have been carried out.

The data were also divided by age: there were 36 pairs of twins under 18 years, 34 pairs aged 18 to 30 years, and 25 pairs aged over 30 years. Two results only, out of 87 comparisons, were found to be significant; relative severity of headaches for the 18 to 30 year olds and relative severity of coughing for those over 30 years. The severities of both were lower for the vitamin $\mathrm{C}$ group but were only just significant at the $5 \%$ level.

Another division of the data was the 51 pairs who stated that they lived together and the 44 pairs who stated that they lived apart. These results are shown in Table 2 and 
TABLE 1. Treatment Means of Summary Cold Variables for Males, Females, and Total Sample: Significance of Difference Between Active and Placebo Groups Indicated

\begin{tabular}{|c|c|c|c|c|c|c|}
\hline & \multicolumn{2}{|c|}{ Males (38 pairs) } & \multicolumn{2}{|c|}{ Females ( 57 pairs) } & \multicolumn{2}{|c|}{ Total (95 pairs) } \\
\hline & Active & Placebo & Active & Placebo & Active & Placebo \\
\hline Incidence & 1.26 & 1.26 & 1.81 & 1.63 & 1.58 & 1.48 \\
\hline Total duration & 8.58 & 8.76 & 12.7 & 12.6 & 11.0 & 11.1 \\
\hline Average duration & 4.37 & 5.08 & 5.72 & 7.26 & $5.18^{*}$ & 6.39 \\
\hline Total severity & 34.4 & 37.8 & 56.7 & 53.3 & 47.8 & 47.1 \\
\hline Average severity & 17.3 & 21.3 & 26.5 & 31.6 & 22.8 & 27.5 \\
\hline Intensity & 2.73 & 3.12 & 3.68 & 3.95 & 3.30 & 3.62 \\
\hline
\end{tabular}

$* \mathbf{P}<0.05$.

TABLE 2. Treatment Means for Twins Living Together and Living Apart

\begin{tabular}{lccccc}
\hline & \multicolumn{2}{c}{ Living together (51 pairs) } & & \multicolumn{2}{c}{ Living apart (44 pairs) } \\
\cline { 2 - 3 } \cline { 6 - 7 } & Active & Placebo & & Active & Placebo \\
\hline Incidence & $1.84^{*}$ & 1.37 & & 1.30 & 1.61 \\
Total duration & $13.7^{*}$ & 9.6 & & $8.0^{*}$ & 12.8 \\
Average duration & 5.46 & 5.42 & & $4.86^{* *}$ & 7.50 \\
Total severity & $57.9^{*}$ & 37.8 & & $36.0^{*}$ & 57.9 \\
Average severity & 23.6 & 22.2 & & $21.9^{*}$ & 33.6 \\
Intensity & 3.36 & 3.24 & & $3.23 \dagger$ & 4.05 \\
\hline *P $<0.05$ & & & & \\
$* * \mathrm{P}<0.01$. & & & & \\
$\dagger \mathrm{P}<0.10$. & & & & & \\
& & & & &
\end{tabular}

reveal a paradoxical effect of the active and placebo treatments. Among the twins living together, those taking vitamin $\mathrm{C}$ had a significantly higher incidence, total duration, and total severity of colds than those taking the placebo. Total severity of sneezing and blocked nose were also greater in the vitamin $\mathrm{C}$ group.

Among the pairs living apart there were nine significant treatment differences but, in contrast to the results from pairs living together, all of these favored the vitamin $\mathrm{C}$ group. Total duration and average severity of colds, total severities of coughing, runny nose and fever, and relative severities of coughing and headache were all found to be significantly lower than in the placebo group.

It seems that the treatment effects observed in the pairs living together and pairs living apart were of similar magnitude but of opposite direction so that when the groups were comoined they canceled each other out. When the pairs living apart and living together were further subdivided by sex, the same effects were apparent in both males and females, although with a drop in significance levels commensurate with the smaller numbers in each cell.

There is no significant association of actual treatment and twins' perception of their treatment $\left(\chi^{2}=3.39\right)$, but, if anything, there was a tendency for twins to guess their treatment group wrongly. Answers to a further question at the end of the trial revealed that, of those who expressed an opinion on the treatment group to which they belonged, most based this opinion on their perceived experience of the number of colds in comparison 
with previous years. However, there was no association between treatment group and number of colds perceived by the subjects $\left(\chi^{2}=3.12\right)$.

It is possible that perception of treatment group and reporting of cold symptoms are, for whatever causal reason, closely associated. To test this possibility, pairs where one twin believed (rightly or wrongly) he was taking the "high dose" and the other believed he was taking the "low dose" were selected for analysis. Only 26 pairs fulfilled these strict criteria and their results were analyzed according to their perceived treatment and regardless of their actual treatment. The results of this analysis (Table 3) reveal very strikingly that the twins who thought they were on "high dose" reported markedly fewer, shorter, less severe, and less intense colds than their cotwins who thought they were on "low dose."

Although there is no overall association between perceived and actual treatment group in the total data, it is possible that there is a nonrandom association in one or both of the subgroups living together or apart. Table 4 shows an almost significant $(\mathrm{P}=0.06)$ proportion of subjects incorrectly guessing their actual treatment in the twins living together, but Table 5 shows no corresponding disproportion of perception between treatment groups in pairs living apart.

TABLE 3. Comparison of Cold Results by Treatment Perception in 26 Pairs With Dichotomous Perception

\begin{tabular}{lcc}
\hline & $\begin{array}{c}\text { Perceived } \\
\text { low dose }\end{array}$ & $\begin{array}{r}\text { Penceived } \\
\text { high dose }\end{array}$ \\
\hline Incidence & $1.96 * *$ & 1.19 \\
Total duration & $16.4^{* * * *}$ & 6.2 \\
Average duration & $6.88^{* *}$ & 3.74 \\
Total severity & $82.7^{* * *}$ & 26.2 \\
Average severity & $35.6^{* * *}$ & 16.6 \\
Intensity & 4.26 & 2.96 \\
\hline
\end{tabular}

**P $<0.01$.

***P $<0.001$.

TABLE 4. Treatment Perception by Actual Treatment of Twins Living Together*

\begin{tabular}{lcccr}
\hline & Low dose & High dose & No idea & Total \\
\hline Active & 20 & 7 & 24 & 51 \\
Placebo & 14 & 17 & 20 & 51 \\
Total & 34 & 24 & 44 & 102 \\
\hline
\end{tabular}

${ }^{*} \chi^{\frac{2}{2}}=5.59, P=0.06$.

TABLE 5. Treatment Perception by Actual Treatment of Twins Living Apart*

\begin{tabular}{lcccc}
\hline & Low dose & High dose & No idea & Total \\
\hline Active & 14 & 13 & 17 & 44 \\
Placebo & 10 & 12 & 22 & 44 \\
Total & 24 & 25 & 39 & 88 \\
\hline
\end{tabular}

$x^{2}=1.35, P=0.51$ 
Personality scores from the EPQ were correlated with cold variables. No significant correlations were observed with extraversion, psychoticism, or the lie scales. However, the six main cold variables and eight of the individual total severity measures all correlated significantly with neuroticism, coefficients being in the range 0.2-0.3.

In the biochemical results, a significant rise in alanine aminotransferase and fall in uric acid levels were observed in the vitamin $\mathrm{C}$ group compared to the placebo group. However, neither change was substantively important. No significant differences between treatment groups in total cholesterol, HDL cholesterol, non-HDL cholesterol, or triglycerides were observed. The biochemical results will be reported in full elsewhere [4].

At the conclusion of the trial, twins were asked whether they had experienced any changes that they considered could possibly be due to the tablets they had taken during the trial. The heterogeneous collection of symptoms reported was evenly distributed between the control and treatment groups and no obvious effects of either vitamin $\mathrm{C}$ or placebo were apparent.

\section{DISCUSSION}

The analyses suggest that the placebo was a good one and that the double-blind design of the experiment was not compromised. Results from the total data do not suggest any effect of a daily $1 \mathrm{~g}$ dose of vitamin $\mathrm{C}$ in preventing the common cold. However, among pairs living together the placebo group reports significantly fewer colds but the opposite effect is observed among those living apart.

Reporting of cold symptoms is intimately related to perception of treatment. Whether this association arises because treatment perception determines reporting or reporting determines perception, or because experience of colds determines both perception and reporting, cannot be decided with these data. The apparent effect of the placebo in preventing colds in the pairs living together may be related to the disproportionately large number in that group who incorrectly guessed their actual treatment. However, there is no significant association between perception and treatment group in the pairs living apart, so it is possible that the observed preventive effect of vitamin $\mathrm{C}$ in this group is real. Among these pairs, the vitamin $\mathrm{C}$ group got $19 \%$ fewer colds, the total duration was $38 \%$ less, total severity $22 \%$ less, and intensity was $20 \%$ less than in the placebo group.

It is also possible that the apparent treatment effect in this group has occurred by chance particularly since there is otherwise a total lack of evidence for the effect of vitamin $C$ in the other groupings of the data. The fact that self-reporting of cold symptoms is strongly related to perception of treatment and to the personality dimension neuroticism suggests that even a small nonrandom distribution of these characters in control and treatment groups could produce a spurious treatment effect.

Comparison of the paired and unpaired analyses of the data showed that, averaged over all cold symptoms, 1.36 times more unrelated subjects than we have used would be required to achieve the same power to test the treatment effect. This is not a great gain in efficiency over the random groups design but must be contrasted with an efficiency factor of 3.85 achieved in the analysis of the biochemical data analyzed from the same experiment [4].

Final conclusions from the experiment must remain unclear. All the most obvious analyses suggest that vitamin $\mathrm{C}$ has had no effect at all in preventing colds. However, it is possible that nonrandom perception of treatment in parts of the sample has hidden a genuine effect of vitamin $\mathrm{C}$ in reducing the incidence, duration, and severity of colds 
by about $20 \%$. Such an effect was observed in the pairs of twins living apart. In either case our results are consistent with those of earlier trials [1-3,5,6,8,10,11,13-16] in suggesting that a Ig daily dose of vitamin $\mathrm{C}$ has, at best, only a modest effect in preventing colds.

\section{ACKNOWLEDGMENTS}

We wish to thank Roche Products, in particular Dr. Michael Henderson, for supplying the vitamin and placebo tablets and for financial support to cover postage costs. We are grateful to Miss Janet Craig and others who took blood specimens and to Dr. J.B. Gibson and Dr. J.D. Mathews for critical comments on the manuscript. Most of all, we are grateful to the twins for their willing cooperation during the course of the trial.

\section{REFERENCES}

1. Anderson TW, Suranyi G, Beaton GH (1972): Vitamin C and the common cold: A double blind trial. Can Med Assoc J 107:503-508.

2. Anderson TW, Reid DBW, Beaton GH (1974): The effect on winter illness of large doses of vitamin C. Can Med Assoc J 111:31-36.

3. Anderson TW, Beaton GH, Corey PN, Spero L (1975): Winter illness and vitamin C: The effect of relatively low doses. Can Med Assoc J 112:823-826.

4. Carr AB, Martin NG, Whitfield JB (1981): Usefulness of the cotwin control design in investigations as exemplified in a study of effects of ascorbic acid on laboratory test results. Clin Chem 27:1469-1470.

5. Coulehan JL, Reisinger KS, Rogers K, Bradley DW (1974): Vitamin C prophylaxis in a boarding school. N Engl J Med 290:6-10.

6. Coulehan JL, Eberhard S, Kapner MSL, Taylor F, Rogers K, Garry P (1976): Vitamin C and acute illness in Navajo schoolchildren. N Engl J Med 295:973-977.

7. Christian JC, Kang KW (1972): Efficiency of human monozygotic twins in studies of blood lipids. Me tabolism 21:691-699.

8. El wood PC, Hughes SJ, Leger AS (1977): A randomized controlled trial of the therapeutic effect of vitamin $\mathrm{C}$ in the common cold. Practitioner 218:133-137.

9. Eysenck HJ, Eysenck SBG (1975): "Manual of the Eysenck Personality Questionnaire (Junior and Adult)." London: Hodder and Stoughton.

10. Karlowski TR, Chalmers TC, Frenkel LD, Kapikian AZ, Lewis TL, Lynch JM (1975): Ascorbid acid for the common cold: A prophylactic and therapeutic trial. JAMA 231:1038-1042.

11. Miller JZ, Nance WE, Norton JA, Wolen RL, Griffith RS, Rose RJ (1977): Therapeutic effect of vitamin C: A cotwin control study. JAMA 237:248-251.

12. Pauling L (1970): "Vitamin C and the Common Cold." San Francisco: W. H. Freeman and Co.

13. Pauling L (1971): The significance of the evidence about ascorbic acid on the common cold. Proc Natl Acad Sci USA 68:2678-2681.

14. Pitt HA, Costrini AM (1979): Vitamin C prophylaxis in marine recruits. JAMA 241:908-911.

15. Practitioner Research Group (1968): Ineffectiveness of vitamin C in treating coryza. Practitioner 200:442-445.

16. Wilson CWM, Loh HS (1973): Common cold and vitamin C. Lancet 1:638-641.

Correspondence: Dr. N.G. Martin, Department of Population Biology, Research School of Biological Sciences, Australian National University, PO Box 475, Canberra City, ACT, 2601, Australia. 